\title{
Discriminating Supersymmetry and Black Holes at the Large Hadron Collider
}

\author{
Arunava Roy* and Marco Cavagliä \\ Department of Physics and Astronomy, University of Mississippi, University, MS 38677-1848, USA
}

(Dated: October 31, 2018)

\begin{abstract}
We show how to differentiate the minimal supersymmetric extension of the standard model from black hole events at the Large Hadron Collider. Black holes are simulated with the CATFISH generator. Supersymmetry simulations use a combination of PYTHIA and ISAJET. Our study, based on event shape variables, visible and missing momenta, and analysis of dilepton events, demonstrates that supersymmetry and black hole events at the LHC can be easily discriminated.

PACS numbers: 04.50.-h, 04.70.Dy, 04.65.+e, 11.30.Pb, 12.60.Jv
\end{abstract}

\section{INTRODUCTION}

At CERN's Large Hadron Collider (LHC) 1] protons will soon collide at an astonishing 800 million times per second to provide experimental evidence for the Higgs 2, 3], supersymmetry (SUSY) [4, 5] or extra dimensions [6, 7, 8]. SUSY is widely considered to be one of the best candidates for physics beyond the Standard Model (SM). It provides an explanation for the Higgs mass problem, a candidate for cold dark matter, and unification of low energy gauge couplings by introducing superpartners to SM fields (see Ref. [9] and references therein). An alternative to SUSY is given by phenomenological extra-dimensional models such as Large Extra Dimensions (LEDs) [6], warped braneworlds [7] or universal extra dimensions [8]. Scenarios with LEDs are specially appealing. In these models, gravity becomes strong at the $\mathrm{TeV}$ scale, where radiative stability is achieved. The fundamental scale of gravity, $M_{\star} \sim 1 \mathrm{TeV}$, is related to the observed Planck scale, $M_{\mathrm{Pl}}$, by the relation $M_{\mathrm{Pl}}^{2} \sim V_{n} M_{\star}^{n+2}$, where $V_{n}$ is the volume of the extra $n$-dimensional space. One of the most astounding consequences of the existence of extra-dimensions would be the production of subatomic Black Holes (BHs) in particle colliders [10, 11] and cosmic ray showers [12]. (For reviews, see Refs. [13].)

The ATLAS [14] and CMS [15] experiments at the LHC are entrusted with the task of studying events with large transverse momentum $\left(P_{T}\right)$, a signature common to both SUSY and extra dimensions. While we wait for these experiments to start collecting data, it is worthwhile to look into means of distinguishing SUSY and extradimensional models [16]. Comparisons of SUSY and universal extra dimensions/little Higgs models in colliders have been investigated by various authors [17]. Discrimination of SUSY and BH events by means of dilepton events was recently discussed by the authors in Ref. [18]. In this paper, we revisit that analysis and extend it to include event shape variables, missing transverse momentum $\not_{T}$ and visible energy. BH and SUSY events are simulated with the BH generator CATFISH [19] and the high-energy event generator PYTHIA [20], respectively. SUSY masses are set with ISAJET [21]. The analysis below will show that SUSY and BH events can be clearly distinguished at the LHC. BH events tend to be more spherical than SUSY events because of the isotropic nature of BH decay. Thus event shape variables, such as sphericity, provide good discriminators. On the contrary, visible energy and $\not_{T}$ are less effective discriminators because of the presence of invisible channels in both SUSY and BH models, which make the amount of $\not_{T}$ comparable in the two scenarios. The dilepton invariant mass is also an excellent discriminator; the SUSY invariant mass shows a sharp cutoff at $\sim 100 \mathrm{GeV}$, which is absent in the $\mathrm{BH}$ model because most of the dileptons originate from uncorrelated events.

The remainder of this paper is organized as follows. In Sect. II and III we briefly review the fundamentals of SUSY and TeV BHs which are needed for our analysis, respectively. Simulations are described in Sect. IV The analysis of visible/missing momentum and event shape variables is presented in Sect. $\mathrm{VA}$, and the discrimination of SUSY and $\mathrm{BH}$ using dileptons is discussed in Sect. VB. Conclusions are presented in Sect. VI,

\footnotetext{
*Electronic address: arunav@olemiss.edu

${ }^{\dagger}$ Electronic address: cavaglia@olemiss.edu
} 


\section{SUPERSYMMETRY AT THE LHC}

The Minimal Supersymmetric extension of the Standard Model (MSSM) [4] is the simplest SUSY model. According to the MSSM, all SM fermions (bosons) must have a bosonic (fermionic) partner. Superpartners have identical masses, charges and quantum numbers of their SM counterparts, differing only in their spin. The MSSM allows for the unification of electromagnetic, weak and strong forces at $M_{G U T} \sim 10^{16} \mathrm{GeV}$. Since we do not observe superpartners of SM particles at low energies, SUSY must be a broken symmetry. The SUSY breaking scale, i.e. the mass scale at which we expect the first SUSY particles to appear, is generally assumed to be around 1 TeV. A method of SUSY breaking which is mediated by gravitational interactions is supergravity (SUGRA). In its minimal version, mSUGRA is determined by a point in the five-dimensional moduli space with parameters:

- $m_{0}$, the common scalar mass at $M_{G U T}$;

- $m_{1 / 2}$, the common gaugino mass at $M_{G U T}$;

- $A_{0}$, the common trilinear coupling at $M_{G U T}$;

- $\tan \beta$, the ratio of the vacuum expectation values of the two Higgs fields;

- $\mu$, the sign of the Higgsino mass parameter.

mSUGRA parameters for five typical LHC points are given in Table \22]. Neutralino $\left(\tilde{\chi}_{i}^{0}\right)$, gluino $(\tilde{g})$ and squark $(\tilde{q})$ masses are determined by $m_{0}$ and $m_{1 / 2}$ as $\tilde{\chi}_{1}^{0} \sim m_{1 / 2} / 2, \tilde{\chi}_{2}^{0} \sim \tilde{\chi}_{1}^{ \pm} \sim m_{1 / 2}, \tilde{g} \sim 3 m_{1 / 2}$ and $m(\tilde{q}) \sim\left(m_{0}{ }^{2}+6 m_{1 / 2}^{2}\right)^{1 / 2}$ [23].

TABLE I: Parameters for the five mSUGRA points discussed in the text. The scalar mass and the gaugino mass are given in $\mathrm{GeV}$.

\begin{tabular}{|c|ccccc|}
\hline LHC point & $m_{0}$ & $m_{1 / 2}$ & $A_{0}$ & $\tan \beta$ & $\mu$ \\
\hline $\mathrm{A}$ & 100 & 300 & 300 & 2.1 & + \\
\hline $\mathrm{B}$ & 400 & 400 & 0 & 2 & + \\
\hline $\mathrm{C}$ & 400 & 400 & 0 & 10 & + \\
\hline $\mathrm{D}$ & 200 & 100 & 0 & 2 & - \\
\hline $\mathrm{E}$ & 800 & 200 & 0 & 10 & + \\
\hline
\end{tabular}

Visible energy, missing transverse momentum and sphericity for the five LHC points of Table 1 are shown in Fig. 1 . where all SUSY processes except SM Higgs production have been implemented. Sparticle production at point D (open blue circles) is higher as squarks and gluinos are lighter. This point is usually taken as the comparison point between the LHC and other experiments, e.g. Tevatron [24] and NLC [25]. For the purposes of our analysis, the difference between the five points is not significant and any of them can be chosen as SUSY benchmark. In the following, we will consider point A. This is justified by the fact that point A allows for SUSY Higgs production [26]. Since BHs may evaporate into Higgs (see Sect. III below), a meaningful comparison of SUSY and BH events requires the presence of the Higgs channel in both models. Moreover, distinguishability of SUSY and BH events must be assessed by minimizing the differences between the two models. Since BH events are characterized by up to several TeV of missing transverse momentum, SUSY points with large $\not_{T}$, such as point $\mathrm{A}$, must be considered.

A symmetry of the MSSM is $R$-parity [4]:

$$
P_{R}=(-1)^{3 B+L+2 s}
$$

where $B(L)$ is the baryon (lepton) number and $s$ is the particle spin. All SM particles have $P_{R}=+1$ whereas their superpartners have $P_{R}=-1$. $R$-parity implies that SUSY particles are always pair produced from SM particles. If $R$-parity is conserved, the endpoint of a SUSY process at the LHC is a state with SM particles and two lightest stable SUSY particles (LSPs), which are generally neutralinos. Being colorless and chargeless, the LSPs escape the detector and are the source of missing transverse momentum, a leading signature of SUSY events. If $R$-parity is not conserved, the missing transverse energy is reduced by the LSP decay. In the following, we will assume that $R$-parity is conserved, in agreement with the MSSM (mSUGRA) scenario.

We end this section with a list of dominant SUSY interactions at LHC point A and the definition of invariant mass. This is important for the following analysis because it enables us to select processes that could serve as potential 

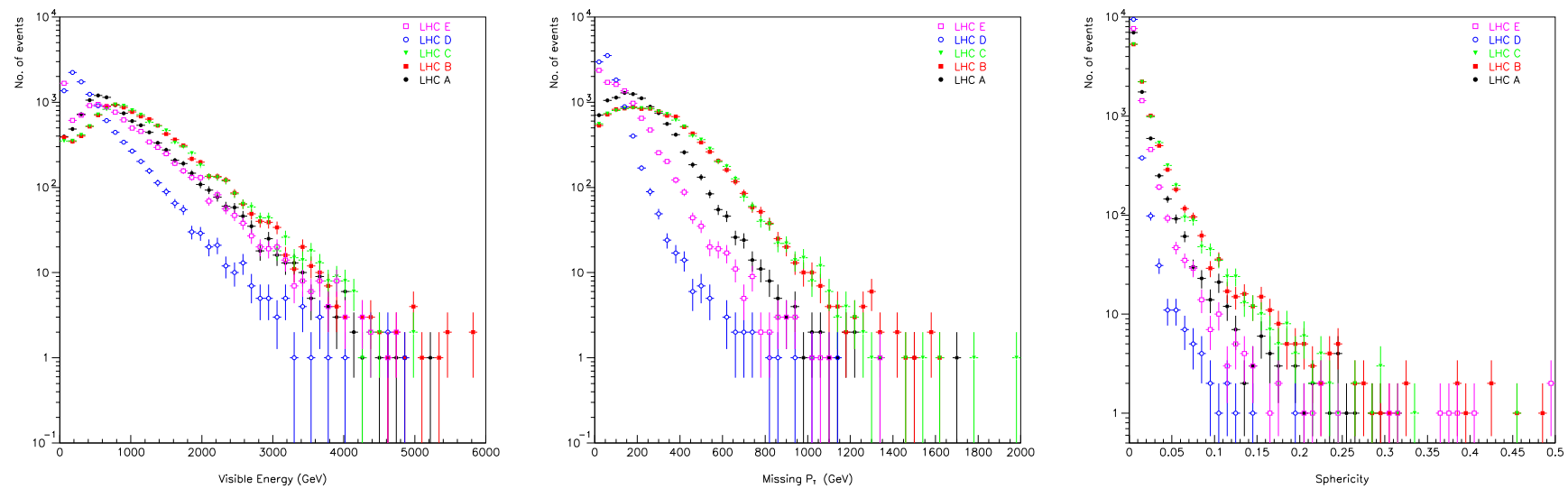

FIG. 1: Comparison of visible energy (left), missing transverse momentum $\not P_{T}$ (middle) and sphericity (right) for 10000 events for the five LHC points of Table【 (A: black filled circles, B: red filled squares, C: green filled triangles, D: blue open circles and E: pink open squares).
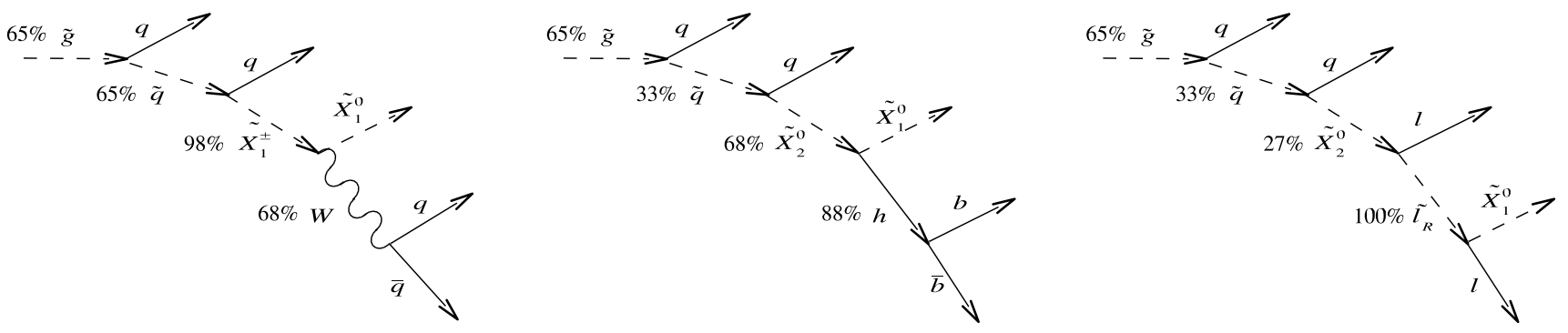

FIG. 2: The top three SUSY decay chains and their branching ratios.

discriminators. The third decay chain in Fig. 2 is specially interesting because it allows the separation of isolated leptons from the hadronic background [27]. The invariant mass is defined as

$$
M_{12}=\sqrt{\left(E_{1}+E_{2}\right)^{2}-\left(\mathbf{p}_{1}+\mathbf{p}_{2}\right)^{2}}=\sqrt{2 \mathbf{p}_{1} \mathbf{p}_{2}(1-\cos \theta)},
$$

where $\theta$ is the angle between the two particles. The method of constructing invariant masses from SUSY decay chains has been traditionally used to calculate sparticle masses [26]. In Sect. VB we will use the invariant mass of isolated dileptons with large $P_{T}$ as a potential SUSY/BH discriminator. (For a review on lepton production at colliders, see Ref. [28].) In the rest frame of the second lightest neutralino, the dilepton invariant mass is

$$
M_{l l}=\left[M_{\tilde{\chi}_{2}^{0}}^{2}+M_{\tilde{\chi}_{1}^{0}}^{2}-2 M_{\tilde{\chi}_{2}^{0}} M_{\tilde{\chi}_{1}^{0}}\left(1+\frac{P_{\tilde{\chi}_{1}^{0}}^{2}}{M_{\tilde{\chi}_{1}^{0}}^{2}}\right)\right]^{1 / 2} .
$$

Since the momentum of the LSP is not constrained, the invariant mass distribution shows an edge at $\sim 100 \mathrm{GeV}$.

\section{BLACK HOLES AT THE LHC}

In LED scenarios, $p p$ collisions at the LHC could produce TeV-mass BHs with characteristic lifetimes of $10^{-25}$ seconds [13]. Numerous studies have focused on BH signatures at the LHC [11] and various Monte Carlo generators 
are available for simulation purposes $\left[19,229,[30]^{1}\right.$. A quick look at BH production at the LHC reveals the following. According to Thorne's hoop conjecture [32], a $\mathrm{BH}$ of mass $M$ is formed when an object is compressed in all directions such that

$$
C<2 \pi R_{s}(M)
$$

where $\mathrm{C}$ is the circumference of the region where the object is compacted into and $R_{s}$ is the Schwarzschild radius for a $\mathrm{BH}$ of mass $M$. An upper limit on the $\mathrm{BH}$ mass is obtained by assuming no gravitational energy loss at formation, corresponding to the black disk (BD) cross-section $\sigma_{B D}=\pi R_{s}^{2}$. A more realistic model assumes that all the CM energy is not available for $\mathrm{BH}$ formation, some being lost as gravitational radiation (see Ref. 33] for a more detailed discussion). To estimate the energy loss, the colliding particles are treated as two Aichelburg-Sexl shock waves [34]; the overlap of the shock waves forms a trapped-surface (TS) which sets a lower limit to the mass of the BH [35]. (For an alternative estimate of the collisional gravitational loss, see Ref. [36].) The cross-section at the LHC involves summing up the contributions from all the initial partons. The cross section in the TS scenario is

$$
\sigma_{p p \rightarrow B H}(s, n)=\sum_{i j} \int_{0}^{1} 2 z d z \int_{x_{m}}^{1} d x \int_{x}^{1} \frac{d x^{\prime}}{x^{\prime}} f_{i}\left(x^{\prime}, Q\right) f_{j}\left(x / x^{\prime}, Q\right) F \sigma_{B D}(x s, n),
$$

where $Q$ is four-momentum transfer squared, $f_{i}(x, Q)$ are the parton distribution functions, $z$ is the normalized impact parameter, and $F$ is a form factor. The cutoff in $x$ is related to the minimum allowed mass of the object, $M_{\min }$, and the fraction of center-of-mass energy trapped in the BH, $y(z)$, by $x_{m}=M_{\min }^{2} /\left[s y^{2}(z)\right]$. TeV BHs may carry electric or color charge and angular momentum. Immediately after formation, they are expected to decay through loss of excess multipole moments (balding phase), gravitational + Hawking radiation 37] (evaporation phase) and final $n$-body decay or remnant production (Planck phase). SM particles are emitted on the brane and can be detected [38]. Since the balding phase is poorly understood, simulations neglect the energy loss in this phase. The description of the evaporation phase is also approximated; since emissivities of rotating BHs are not known for all fields, $\mathrm{BH}$ generators use greybody factors for non-rotating BHs [39, 40]. In CATFISH, the total decay multiplicity is [19]

$$
N=\frac{(n+1) S}{4 \pi} \frac{\sum_{i} c_{i} \mathcal{R}_{i} \Gamma_{\mathcal{R}_{i}}}{\sum_{j} c_{j} \mathcal{P}_{j} \Gamma_{\mathcal{P}_{j}}}
$$

where $c_{i}$ are the degrees of freedom of species $i, \Gamma_{\mathcal{P}_{i}}$ and $\Gamma_{\mathcal{R}_{i}}$ are the relative emissivities of Ref. [40], $S$ is the initial entropy of the $\mathrm{BH}$, and $\mathcal{P}_{s}$ and $\mathcal{R}_{s}$ are spin-dependent normalization factors. A more detailed discussion of the evaporation and Planck phases of TeV BHs can be found in Refs. [13].

Dilepton production in BH events differ greatly from dilepton production in the MSSM. Unlike SUSY, there is no single process of dilepton production; dileptons are either produced by the BH directly or by the decay of heavier particles such as the $Z_{0}$ boson, $t \bar{t}$ pairs of a combination of the two. Therefore, the BH dilepton invariant mass does not show a sharp cut-off at high energy.

\section{EVENT SIMULATIONS}

SUSY simulations are carried out using a combination of ISAJET and PYTHIA, with the former generating the mass spectrum. BH simulations are carried out using the CATFISH Monte Carlo generator. The setup for each simulation is summarized below:

- SUSY:

- The MSSM mass spectrum is generated with ISAJET (ver. 7.75);

- The mass spectra in SLHA format is fed into PYTHIA;

- All SUSY processes except SM Higgs production are simulated;

\footnotetext{
${ }^{1}$ Recently, a new BH generator (BlackMax) appeared in the literature [31]. BlackMax is supposed to include BH rotational effects. However, the gravitational loss for rotating BHs is artificially set to zero. Since the energy loss due to gravitons is enhanced by rotation and extra-dimensional effects, and cannot be neglected, BlackMax results for rotating BHs should not be trusted.
} 
- Unstable SM particles and sparticles are hadronized or decayed with PYTHIA.

- BHs:

- The cross section for a BH event is calculated in the center-of-mass frame;

- The initial BH mass is sampled from the differential cross section;

- The BH is decayed through Hawking mechanism and final $n$-body event (or remnant);

- Unstable quanta are hadronized or decayed with PYTHIA.

The benchmark model for SUSY is LHC point A. The parameters for the BH benchmark model are fundamental Planck scale $M_{\star}=1 \mathrm{TeV}$, minimum $\mathrm{BH}$ mass $M_{\min }=2 \mathrm{TeV}$, classical-to-quantum threshold $Q_{\min }=1 \mathrm{TeV}$, six extra-dimensions $(n=6)$ and two-body final decay $\left(n_{p}=2\right)$. Particles produced in the initial-radiation phase are removed by imposing $P_{T}$ cuts of $5 \mathrm{GeV}$ and $15 \mathrm{GeV}$ for leptons and photons+hadrons, respectively [19].

\section{EVENT ANALYSIS}

In this section, we first use event shape variables to discriminate SUSY and BHs models. We then complement these results by looking at isolated dilepton events. The salient features of this analysis is that $\mathrm{BH}$ events tend to be more spherical than SUSY events due to the spherical nature of the Hawking radiation. This is specially evident for high-mass BHs. The formation of a stable $\mathrm{BH}$ remnant at the end of the evaporation phase also helps to discriminate MSSM and BH events because of the large amount of energy which is carried away by the remnant. Isolated dilepton events provide a further powerful means to distinguish the two models. This is due to the fact that leptons are rarely emitted by BHs (the hadron-to-lepton ratio is approximately 5:1) and are uncorrelated; they can be emitted at any angle w.r.t. beam axis, whereas SUSY dileptons typically originate from a single decay chain.

\section{A. $\quad P_{T}$ and event shape variables}

Figure 3 shows visible energy, missing transverse momentum, and transverse momentum of leptons and hadrons+photons for 10,000 MSSM and BH benchmark events. Even in the absence of a BH remnant, the amount of visible energy and $\not_{T}$ is comparable for the two scenarios. This is due to the presence of invisible channels in both models: the LSP for SUSY and neutrinos+gravitons for the BH. The flavor of the decay products is a better discriminator. MSSM interactions do not produce leptons with energy above the $\mathrm{TeV}$ since isolated leptons are produced by the decay of sparticles with typical energy of less than a few hundred GeV. On the contrary, quanta produced in the BH decay are characterized by an average energy $E \sim M / N$, where the multiplicity $N$ is less than 10 for typical BHs at the LHC. Since Hawking evaporation does not distinguish leptons from hadrons, hard leptons with energy up to several $\mathrm{TeV}$ are likely to be produced during the $\mathrm{BH}$ decay. This suggests that isolated leptons may provide a powerful means to discriminate the two models. This is indeed the case, as we shall see in the next section.

The four plots in the left panel of Fig. 4 show how variations in the BH Planck phase affect the observables of Fig. 3 , The plots compare two- and four-body decays to the formation of a $\mathrm{BH}$ remnant $\left(n_{p}=0\right)$. By the time the remnant has formed, the $\mathrm{BH}$ is expected to have shed electric and color charges. (See, however, Ref. [41] for an alternative scenario.) If this is the case, the $\mathrm{BH}$ remnant is undetectable and a source of missing transverse momentum in addition to neutrinos and gravitons which are emitted during the Hawking evaporation phase. This leads to a larger difference in $\not_{T}$ between the MSSM and BH models. The visible transverse momentum in hadrons+photons is sensibly reduced in the presence of a $\mathrm{BH}$ remnant; the latter carries away energy which otherwise would have been emitted in visible channels (mostly hadrons) during the $\mathrm{BH}$ decay phase. It is interesting to note that the amount of transverse momentum in the leptonic channel is essentially unaffected by the presence of a BH remnant. This is due to the fact that leptons are rarer than hadrons in the $\mathrm{BH}$ decay phase; variations in the energy distribution of the leptonic channel are thus suppressed compared to the hadronic channel. Changes in the number of final Planckian hard quanta do not produce significant differences in the distributions; more quanta of lower energy behave statistically like less quanta with higher energy. Provided that the BH decays at the end of the Hawking phase, it is thus safe to set the number of Planckian quanta to $n_{p}=2$ or $n_{p}=4$, although BHs may decay in different numbers of particles on a event-to-event basis. Variations in the classical-to-quantum threshold $Q_{\min }$ are also not expected to cause significant differences in 

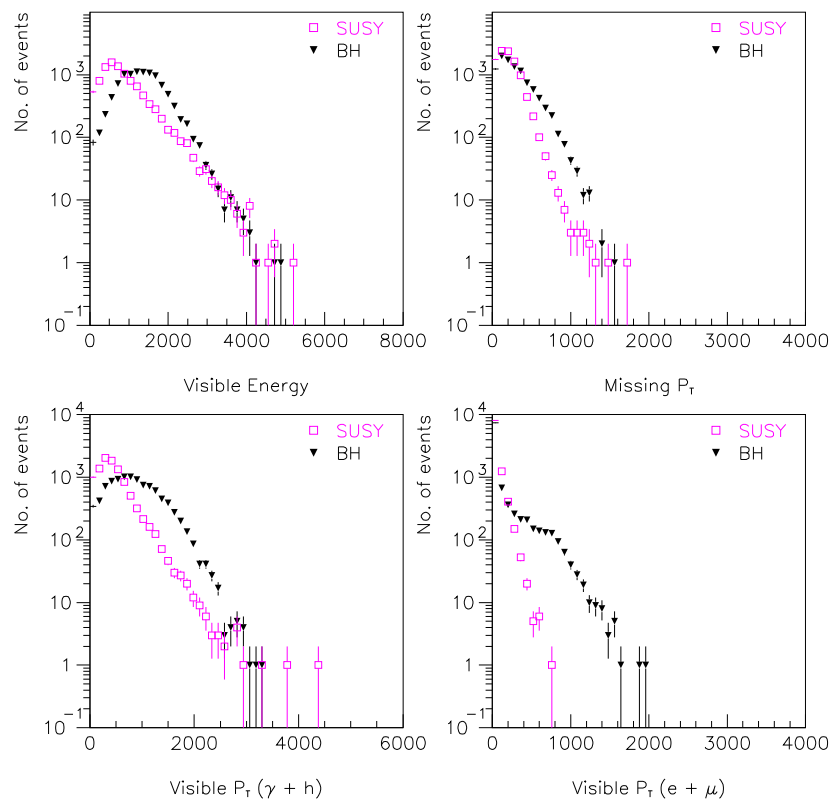

FIG. 3: Comparison of 10,000 SUSY and BH benchmark events at the LHC. Visible energy and missing transverse momentum $\not_{T}$ (top panels) are comparable due to the presence of invisible channels in both models. Leptons with large transverse momentum provide instead an effective discriminator (bottom right panel).

the energy/momentum distributions. A higher threshold increases the emission in the Planck phase while decreasing Hawking radiation. Since these phases differs only in relative greybody factors, the effect is too small to be detected.

The four plots in the right panel of Fig. 4 show visible energy, $\not_{T}$, and visible transverse momenta of leptons and hadrons+photons for different values of the fundamental Planck scale. Higher values of $M_{\star}$ lead to more massive BHs, i.e. higher multiplicity and more energetic quanta. This causes a significant increase in missing and visible momenta. If the value of the fundamental Planck scale happens to be large, $\mathrm{BHs}$ are likely to be found and easily distinguished from SUSY through detection of highly-energetic isolated leptons and hadronic jets. Missing transverse momentum of several $\mathrm{TeV}$ would also be observed.

Event shape variables such as sphericity and $2^{\text {nd }}$ Fox-Wolfram moment can be used to complement the above analysis. BH events are more spherical because of the nature of Hawking radiation and the "jetty" nature of SUSY decays. Formation of a BH remnant and high values of the fundamental scale lead to significant higher sphericity than SUSY (top panels of Fig. (5). The $2^{\text {nd }}$ Fox-Wolfram moment (bottom panels of Fig. (5) is stable versus changes in the $\mathrm{BH}$ Planck phase and provides a good MSSM/BH discriminator. $\mathrm{BH}$ models with higher $M_{\star}$ can be differentiated more easily from the MSSM.

Similar conclusions can be reached by looking at jet masses and number of jets. The MSSM generates more and lighter jets than the $\mathrm{BH}$ model due to copious production of quarks (Fig. (6)). The difference is again specially significant for high values of $M_{\star}$ and in the presence of $\mathrm{BH}$ remnants. Absence of sub- $Q_{\min }$ hard jets could provide strong evidence for BH remnant production. (See the suppression of heavy jets below the classical-to-quantum threshold $Q_{\min }=2$ $\mathrm{TeV}$ in the top leftmost panel of Fig. 6.)

\section{B. Event analysis using high- $P_{T}$ dileptons}

The use of isolated dileptons as SUSY signature has been extensively discussed in the literature [26, 42]. Although their production is not as high as colored particles, high-energy isolated leptons provide a cleaner environment by allowing the removal of the QCD background. Moreover, since most of BHs produced at the LHC are expected to be very light, multi-particle analysis may not provide the most effective discriminators [19, 43]. The study of leptonic final states alleviates this problem. 

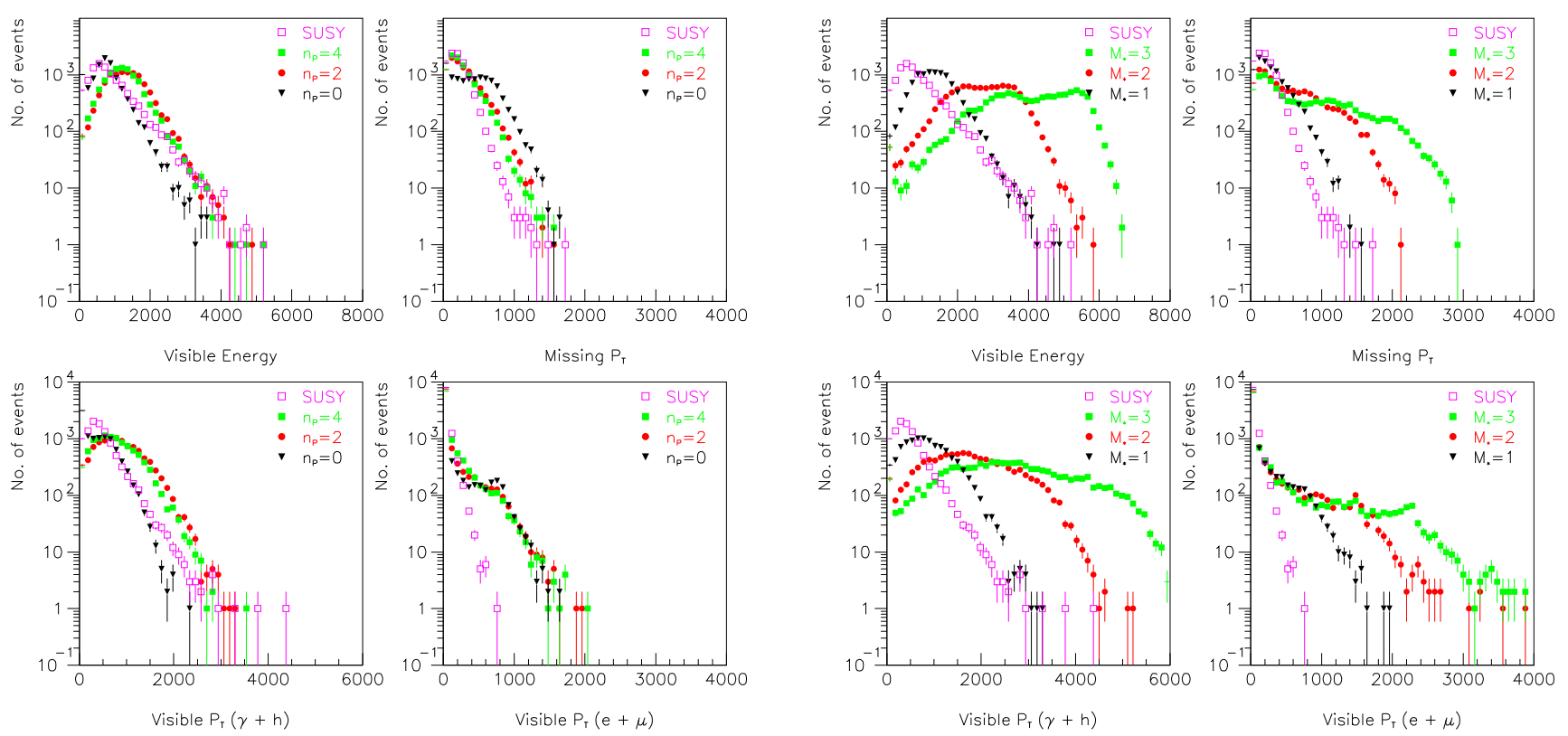

FIG. 4: Distribution of visible energy, $\not P_{T}$ and transverse momenta of leptons and hadrons+photons. SUSY plots are shown as pink open squares. The four plots in the left panel show the effect of different decay modes in the Planck phase of tendimensional BHs: remnant formation $\left(n_{p}=0\right.$, filled black triangles), two-body decay $\left(n_{p}=2\right.$, filled red circles) and four-body decay $\left(n_{p}=4\right.$, filled green squares). The fundamental Planck scale is $M_{\star}=1 \mathrm{TeV}$. The four plots in the right panel show the effect of varying the fundamental Planck scale: $M_{\star}=1 \mathrm{TeV}$ (filled black triangles), $M_{\star}=2 \mathrm{TeV}$ (filled red circles) and $M_{\star}=3$ $\mathrm{TeV}$ (filled green squares). The ten-dimensional BHs decay in two hard quanta at the end of the evaporation phase.

The dominant MSSM interaction for opposite-sign, same-flavor (OSSF) dileptons at LHC point A is [27]

$$
\begin{aligned}
\tilde{\chi}_{2}^{0} \rightarrow l^{ \pm} & \tilde{l} \\
& \stackrel{\rightarrow}{ } l^{\mp} \tilde{\chi}_{1}^{0}
\end{aligned}
$$

with a branching ratio of $27 \%$. The maximum dilepton invariant mass for this interaction is

$$
M_{l l}^{\max }=m_{\tilde{\chi}_{2}^{0}}\left[\left(1-\frac{m_{\tilde{l}}^{2}}{m_{\tilde{\chi}_{2}^{0}}^{2}}\right)\left(1-\frac{m_{\tilde{\chi}_{1}^{0}}^{2}}{m_{\tilde{l}}^{2}}\right)\right]^{1 / 2} \sim 100 \mathrm{GeV} .
$$

The background for this process is due to SM decays of $W, Z$ bosons and top quarks. This background can be removed by applying suitable cuts on transverse momentum and sphericity of the leptons [22]:

- $P_{T l} \geq 15 \mathrm{GeV},\left|\eta_{l}\right|<2.5$

- Isolation cut, $\sum_{l} P_{T l}<7 \mathrm{GeV}$ in a cone of $R=0.2$,

where $P_{T l}$ is the transverse momentum of the leptons, $R=\sqrt{\Delta \eta^{2}+\Delta \phi^{2}}, \eta=-\ln [\tan (\theta / 2)]$ is the pseudorapidity, and $\phi$ and $\theta$ are the azimuthal and polar angles of the lepton w.r.t. beam axis, respectively.

Isolated leptons in $\mathrm{BH}$ decays come directly from the $\mathrm{BH}$ itself, from the decay of $Z_{0}$ bosons and top quarks, or from a combination of the two. Since the branching ratio of $Z_{0}$ into leptons is small, $\Gamma\left(l^{+} l^{-}\right) / \Gamma_{\text {tot }} \sim 0.034$ [44], and the decay of top quarks into leptons is rare [45], production of OSSF dileptons is less frequent in the BH model than in the MSSM. our analysis shows that an OSSF dilepton event occurs approximately every $100 \mathrm{BH}$ and 20 SUSY events, with a $\sim 1: 5$ ratio of BH-to-SUSY dilepton events at fixed luminosity.

Figure 7 shows the dilepton invariant mass distribution for the MSSM (shaded pink plot) and the BH model with final two- and four-body decay (left and right panels, respectively). As expected, the SUSY distribution shows a sharp 

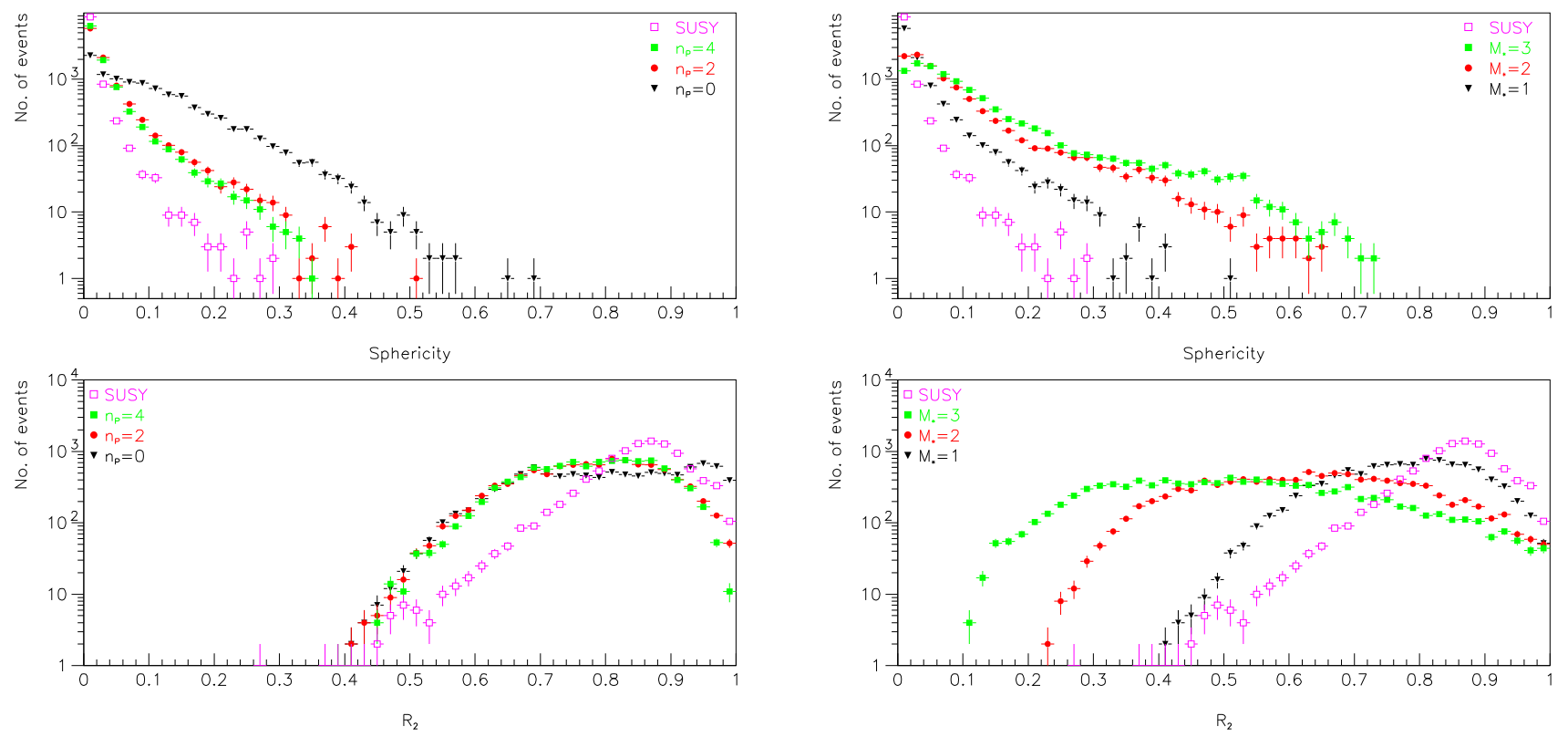

FIG. 5: Sphericity (top panels) and $2^{\text {nd }}$ Fox-Wolfram moment (bottom panels) for 10,000 BH and MSSM events (pink open squares). The left panels show the effect of different Planckian decay modes: BH remnant (filled black triangles), two-body decay (filled red circles) and four-body decay (filled green squares). The fundamental scale is $M_{\star}=1 \mathrm{TeV}$ and the number of extra dimensions is six. The right panels show the effect of different fundamental scales: $M_{\star}=1 \mathrm{TeV}$ (filled black triangles), $2 \mathrm{TeV}$ (filled red circles) and $3 \mathrm{TeV}$ (filled green squares). The ten-dimensional BHs decay in two quanta at the end of the Hawking phase.
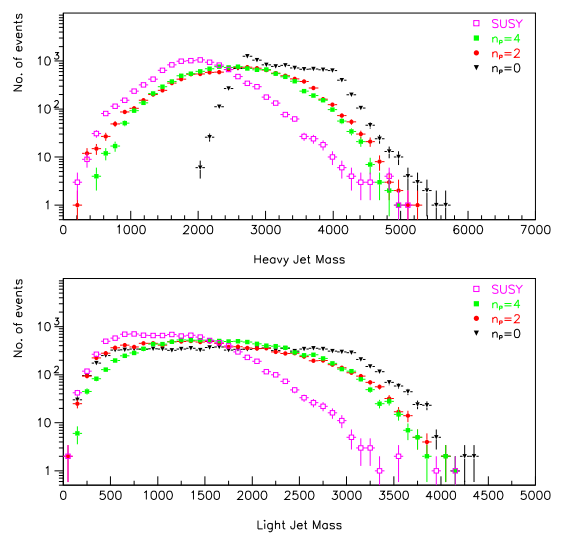

FIG. 6: Light and heavy jet masses (four leftmost panels) and number of jets (right panels) for 10,000 BH and MSSM events. Symbols are like in previous figures.

edge at $\sim 100 \mathrm{GeV}[22]$. The $\mathrm{BH}$ invariant mass distribution is characterized by a peak at $\sim 90 \mathrm{GeV}$ and a long tail up to energy of several $\mathrm{TeV}$. The peak is due to dileptons events produced from the decay of $Z_{0}$ bosons, the dominant channel for OSSF dilepton production in BH. The tail is originated by uncorrelated lepton pairs emitted directly by the $\mathrm{BH}$ or in top quark decays. The leptons are hard and the reconstructed dilepton mass can have super-TeV values. The BH invariant mass for the two-body Planckian decay shows a second, smaller peak at $\sim 1 \mathrm{TeV}$. This occurs because the $\mathrm{BH}$ at the end of the Hawking phase may decay in two OSSF leptons, leading to a reconstructed dilepton invariant mass equal to $Q_{\min }$. If the Planckian decay is a four-body process, the $\mathrm{BH}$ mass at the end of the Hawking evaporation is distributed among four quanta. This produces a lower, smoother reconstructed invariant mass. 

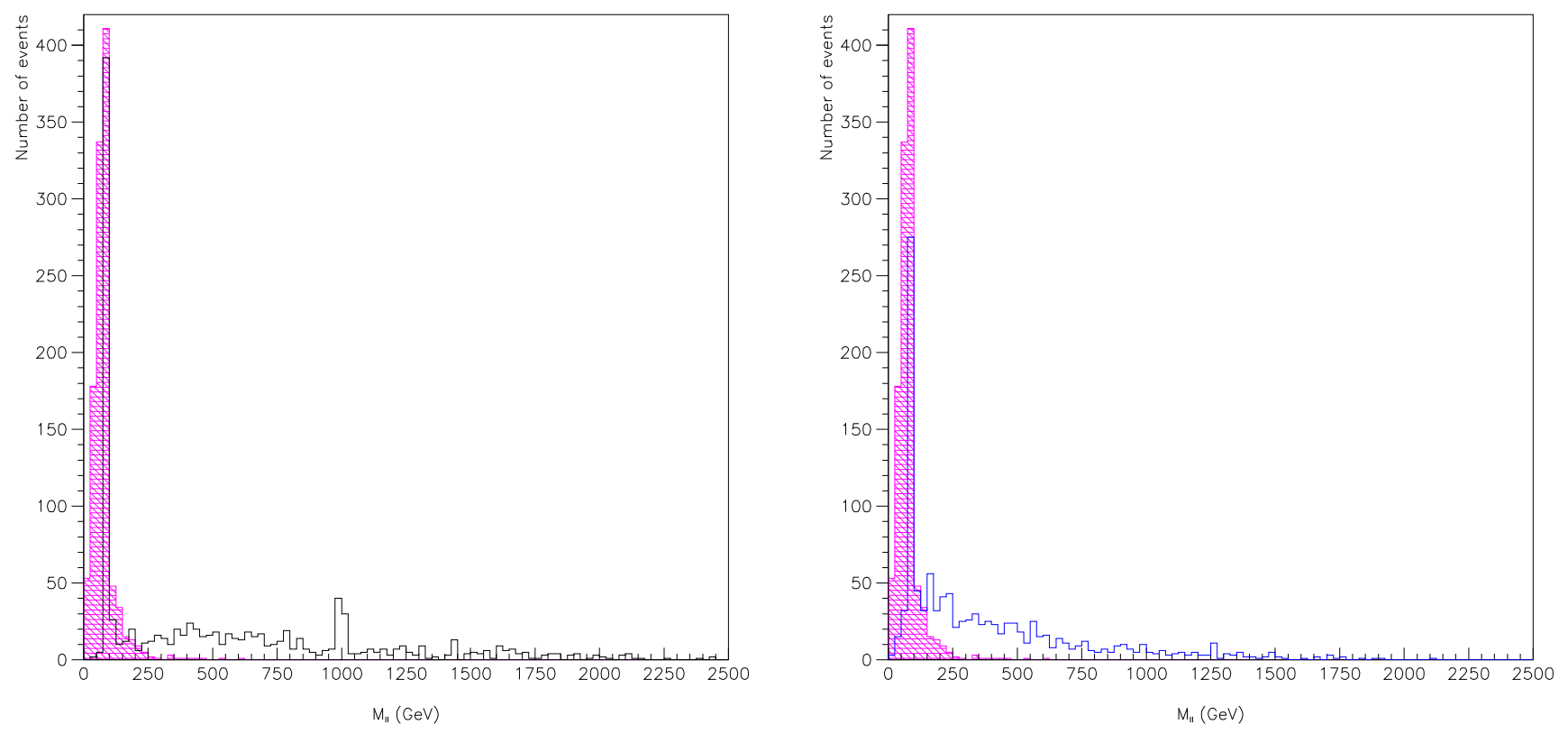

FIG. 7: Invariant mass distribution (in GeV) for 1000 SUSY and BH OSSF dilepton events. The SUSY distribution (shaded pink histogram) shows the typical endpoint due to the presence of the LSP. The high- $P_{T}$ tail of the BH distribution is originated by uncorrelated lepton pairs emitted during the Hawking evaporation phase. The final BH decay is in two-quanta (left panel) or four-quanta (right panel).

The number of isolated, high- $P_{T}$ leptons can also be used to complement the dilepton analysis (left panel of Fig. 8). SUSY events are capable of producing up to five isolated leptons from the cascade decay of heavy sparticles. Events with $\tilde{\chi}_{2}^{0} \tilde{\chi}_{2}^{0}$ or $\tilde{\chi}_{1}^{ \pm} \tilde{\chi}_{2}^{0}$ may produce four or three isolated leptons, respectively [46]. On the contrary, events with three or more isolated leptons are very suppressed in BH decays at the LHC energy. Although multilepton events are rare, there is very little background and they could be effectively used to distinguish the MSSM and the BH model. Other effective discriminators can be constructed by looking at dilepton events with same sign and/or opposite-flavor leptons. The "democratic" nature of the BH decay makes all dilepton events roughly equally probable, whereas the MSSM favors same-flavor dileptons. Presence of hard opposite-flavor leptons is a clear indication of BH decay (right panel of Fig. 8). Our analysis shows that $73 \%$ of SUSY dilepton events are OSSF, compared to only $50 \%$ in the BH model. Conversely, opposite-flavor events are twice more frequent in the BH model (40\%) compared to the MSSM $(21 \%)$.

\section{CONCLUSIONS}

We have discussed and compared the signatures of the MSSM and the TeV-BH model at the LHC. A thorough analysis of event-shape variables and dilepton events has shown that it is possible to distinguish the two models. $\mathrm{BH}$ events are characterized by higher sphericity than SUSY processes. If a $\mathrm{BH}$ remnant is formed at the end of the evaporation phase, missing $P_{T}$ and heavy jet mass are effective signatures to discriminate $\mathrm{BH}$ formation from the MSSM. Although event-shape variables alone cannot unequivocally discriminate between SUSY and BHs, their knowledge may prove useful when combined with the analysis of the leptonic channel. Isolated dileptons could provide the "smoking gun" for detecting BHs at the LHC. The BH dilepton invariant mass shows a tail at high energy which is absent in the SM or MSSM. This analysis can be further strengthened by looking at the number and flavor of isolated leptons. 

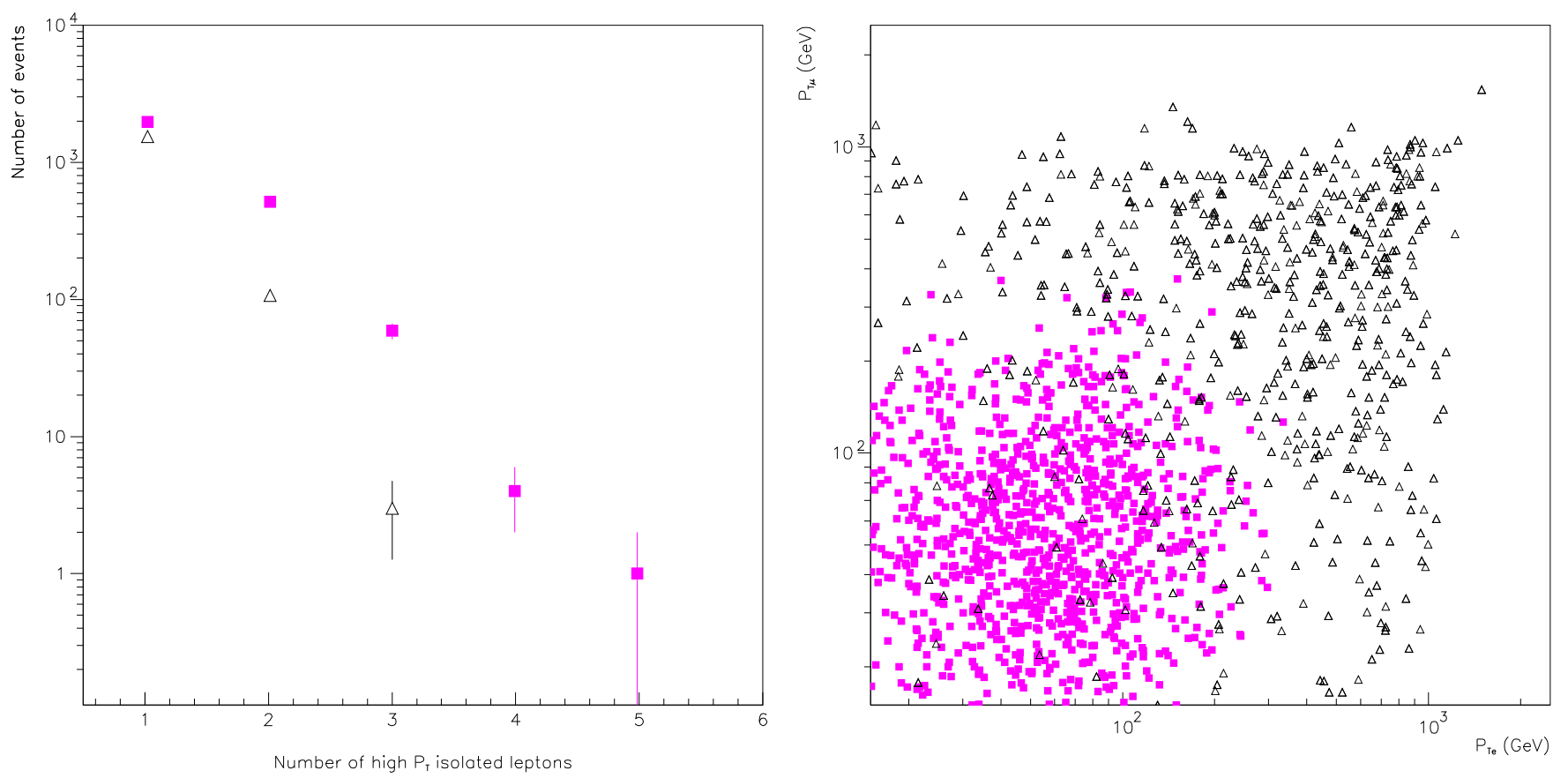

FIG. 8: Left Panel: Histogram of the number of events with high- $P_{T}$ leptons for 10,000 MSSM (pink filled squares) and BH interactions (black open triangles). The number of BH events with three isolated leptons is smaller than the number of SUSY events by a factor of $\sim 20$. The probability of producing BH events with four or more leptons is virtually zero. Right Panel: $P_{T}$ scatter plot for $\sim 1000$ isolated opposite-flavor dilepton events for SUSY (pink filled squares) and BHs (black open triangles). $\mathrm{BH}$ leptons are harder than SUSY leptons and show a larger spread in $P_{T}$.

\section{Acknowledgments}

The authors thank Lucien Cremaldi, Alakabha Datta, Romulus Godang, David Sanders and Don Summers for fruitful discussions, Peter Skands for help with SLHA input file and Xerxes Tata for help on ISAJET. This work was supported (in part) by U.S. DoE contract DE-FG05-91ER40622.

[1] The CERN website, www.cern.ch

[2] P. W. Higgs, Phys. Lett. 12, 132 (1964);

F. Englert and R. Brout, Phys. Rev. Lett. 13, 321 (1964);

G. S. Guralnik, C. R. Hagen and T. W. B. Kibble, Phys. Rev. Lett. 13, 585 (1964);

P. W. Higgs, Phys. Rev. 145, 1156 (1966);

A. Djouadi, arXiv:hep-ph/0503172

[3] "ATLAS TDR on Physics performance, Vol II, Chap. 19," Higgs Bosons (1999);

A. Drozdetskiy [CMS and ATLAS Collaborations], "The standard model Higgs boson at LHC: Recent developments," CERN CMS Note, CERN-CMS-CR-2007-022 (2007);

D. Kcira, arXiv:0710.1957 [hep-ex].

[4] J. Wess and B. Zumino, Phys. Lett. B 49, 52 (1974);

S. Weinberg, "The quantum theory of fields. Vol. 3: Supersymmetry," Cambridge, UK: Univ. Pr. (2000) and references therein.

[5] H. Baer, C. h. Chen, F. Paige and X. Tata, Phys. Rev. D 52, 2746 (1995) arXiv:hep-ph/9503271;

H. Baer, C. h. Chen, F. Paige and X. Tata, Phys. Rev. D 53, 6241 (1996) arXiv:hep-ph/9512383;

F. E. Paige, arXiv:hep-ph/9801254

[6] N. Arkani-Hamed, S. Dimopoulos and G. R. Dvali, Phys. Lett. B 429, 263 (1998) arXiv:hep-ph/9803315;

I. Antoniadis, N. Arkani-Hamed, S. Dimopoulos and G. R. Dvali, Phys. Lett. B 436, 257 (1998) arXiv:hep-ph/9804398;

N. Arkani-Hamed, S. Dimopoulos and G. R. Dvali, Phys. Rev. D 59, 086004 (1999) arXiv:hep-ph/9807344.

[7] L. Randall and R. Sundrum, Phys. Rev. Lett. 83, 3370 (1999) arXiv:hep-ph/9905221; 
L. Randall and R. Sundrum, Phys. Rev. Lett. 83, 4690 (1999) arXiv:hep-th/9906064.

[8] T. Appelquist, H. C. Cheng and B. A. Dobrescu, Phys. Rev. D 64, 035002 (2001) arXiv:hep-ph/0012100;

J. A. R. Cembranos, J. L. Feng and L. E. Strigari, Phys. Rev. D 75, 036004 (2007) arXiv:hep-ph/0612157.

[9] S. P. Martin, arXiv:hep-ph/9709356

M. E. Peskin, arXiv:0801.1928 [hep-ph].

[10] P. C. Argyres, S. Dimopoulos and J. March-Russell, Phys. Lett. B 441, 96 (1998) arXiv:hep-th/9808138;

T. Banks and W. Fischler, arXiv:hep-th/9906038.

S. Dimopoulos and G. Landsberg, Phys. Rev. Lett. 87, 161602 (2001) arXiv:hep-ph/0106295;

S. B. Giddings and S. D. Thomas, Phys. Rev. D 65, 056010 (2002) arXiv:hep-ph/0106219;

E. J. Ahn, M. Cavaglià and A. V. Olinto, Phys. Lett. B 551, 1 (2003) arXiv:hep-th/0201042.

[11] E. J. Ahn and M. Cavaglià, Gen. Rel. Grav. 34, 2037 (2002) arXiv:hep-ph/0205168;

V. P. Frolov and D. Stojkovic, Phys. Rev. Lett. 89, 151302 (2002) arXiv:hep-th/0208102;

V. P. Frolov and D. Stojkovic, Phys. Rev. D 66, 084002 (2002) arXiv:hep-th/0206046;

M. Cavaglià, S. Das and R. Maartens, Class. Quant. Grav. 20, L205 (2003) arXiv:hep-ph/0305223;

A. Chamblin, F. Cooper and G. C. Nayak, Phys. Rev. D 69, 065010 (2004) arXiv:hep-ph/0301239;

M. Cavaglià and S. Das, Class. Quant. Grav. 21, 4511 (2004) arXiv:hep-th/0404050;

A. Chamblin, F. Cooper and G. C. Nayak, Phys. Rev. D 70, 075018 (2004) arXiv:hep-ph/0405054;

C. M. Harris, M. J. Palmer, M. A. Parker, P. Richardson, A. Sabetfakhri and B. R. Webber, JHEP 0505, 053 (2005) arXiv:hep-ph/0411022;

J. Tanaka, T. Yamamura, S. Asai and J. Kanzaki, Eur. Phys. J. C 41, 19 (2005) arXiv:hep-ph/0411095;

B. Webber, In the Proceedings of 33rd SLAC Summer Institute on Particle Physics (SSI 2005): Gravity in the Quantum World and the Cosmos, Menlo Park, California, 25 Jul - 5 Aug 2005, pp T030 arXiv:hep-ph/0511128;

L. Lonnblad, M. Sjodahl and T. Akesson, JHEP 0509, 019 (2005) arXiv:hep-ph/0505181;

J. L. Hewett, B. Lillie and T. G. Rizzo, Phys. Rev. Lett. 95, 261603 (2005) arXiv:hep-ph/0503178;

G. C. Nayak and J. Smith, Phys. Rev. D 74, 014007 (2006) arXiv:hep-ph/0602129;

H. Stoecker, Int. J. Mod. Phys. D 16, 185 (2007) arXiv:hep-ph/0605062;

G. L. Alberghi, R. Casadio, D. Galli, D. Gregori, A. Tronconi and V. Vagnoni, arXiv:hep-ph/0601243;

B. Koch, M. Bleicher and H. Stoecker, J. Phys. G 34, S535 (2007) arXiv:hep-ph/0702187;

D. M. Gingrich, JHEP 0711, 064 (2007) arXiv:0706.0623 [hep-ph]].

[12] J. L. Feng and A. D. Shapere, Phys. Rev. Lett. 88, 021303 (2002) arXiv:hep-ph/0109106;

A. Ringwald and H. Tu, Phys. Lett. B 525, 135 (2002) arXiv:hep-ph/0111042;

L. A. Anchordoqui, J. L. Feng, H. Goldberg and A. D. Shapere, Phys. Rev. D 65, 124027 (2002) arXiv:hep-ph/0112247;

E. J. Ahn, M. Ave, M. Cavaglià and A. V. Olinto, Phys. Rev. D 68, 043004 (2003) arXiv:hep-ph/0306008;

J. I. Illana, M. Masip and D. Meloni, Phys. Rev. D 72, 024003 (2005) arXiv:hep-ph/0504234;

V. Cardoso, M. C. Espirito Santo, M. Paulos, M. Pimenta and B. Tome, Astropart. Phys. 22, 399 (2005) arXiv:hep-ph/0405056;

E. J. Ahn, M. Cavaglià and A. V. Olinto, Astropart. Phys. 22, 377 (2005) arXiv:hep-ph/0312249;

A. Cafarella, C. Coriano and T. N. Tomaras, JHEP 0506, 065 (2005) arXiv:hep-ph/0410358;

E. J. Ahn and M. Cavaglià, Phys. Rev. D 73, 042002 (2006) arXiv:hep-ph/0511159;

M. Cavaglià and A. Roy, Phys. Rev. D 76, 044005 (2007) arXiv:0707.0274 [hep-ph]].

[13] M. Cavaglià, Int. J. Mod. Phys. A 18, 1843 (2003) arXiv:hep-ph/0210296;

R. Emparan, Black hole production at a TeV, arXiv:hep-ph/0302226;

S. Hossenfelder, What black holes can teach us, arXiv:hep-ph/0412265;

P. Kanti, Int. J. Mod. Phys. A 19, 4899 (2004) arXiv:hep-ph/0402168];

G. Landsberg, J. Phys. G 32, R337 (2006) arXiv:hep-ph/0607297.

[14] ATLAS Collaboration, W.W. Armstrong et al., Technical Proposal, CERN/LHCC 94-43, LHCC/P2, 15, December 1994.

[15] CMS Collaboration, G.L. Bayatian et al., Technical Proposal, CERN/LHCC 94-38, LHCC/P1, 15 , December 1994.

[16] V. Buescher, M. Carena, B. Dobrescu, S. Mrenna, D. Rainwater and M. Schmitt, arXiv:hep-ph/0608322

[17] T. G. Rizzo, Phys. Rev. D 64, 095010 (2001) arXiv:hep-ph/0106336;

C. Macesanu, C. D. McMullen and S. Nandi, Phys. Rev. D 66, 015009 (2002) arXiv:hep-ph/0201300;

A. Datta, K. Kong and K. T. Matchev, Phys. Rev. D 72, 096006 (2005) [Erratum-ibid. D 72, 119901 (2005)] arXiv:hep-ph/0509246;

M. Battaglia, A. K. Datta, A. De Roeck, K. Kong and K. T. Matchev, In the Proceedings of 2005 International Linear Collider Workshop (LCWS 2005), Stanford, California, 18-22 Mar 2005, pp 0302 arXiv:hep-ph/0507284;

M. Battaglia, A. Datta, A. De Roeck, K. Kong and K. T. Matchev, JHEP 0507, 033 (2005) arXiv:hep-ph/0502041];

P. Konar and P. Roy, Phys. Lett. B 634, 295 (2006) arXiv:hep-ph/0509161.

[18] A. Roy and M. Cavaglià, arXiv:0710.5490 [hep-ph].

[19] M. Cavaglià, R. Godang, L. Cremaldi and D. Summers, Comput. Phys. Commun. 177, 506 (2007) arXiv:hep-ph/0609001; M. Cavaglià, R. Godang, L. M. Cremaldi and D. J. Summers, JHEP 0706, 055 (2007) arXiv:0707.0317[hep-ph]].

[20] T. Sjostrand, S. Mrenna and P. Skands, JHEP 0605, 026 (2006) arXiv:hep-ph/0603175; See also: http://projects.hepforge.org/pythia6.

[21] F. E. Paige, S. D. Protopopescu, H. Baer and X. Tata, arXiv:hep-ph/0312045.

[22] A. Bartl et al., In the Proceedings of 1996 DPF / DPB Summer Study on New Directions for High-Energy Physics (Snowmass 96), Snowmass, Colorado, 25 Jun - 12 Jul 1996, pp SUP112. 
[23] M. Dittmar, arXiv:hep-ex/9901004.

[24] R. Arnowitt and P. Nath, "Prospects for observation of supersymmetry at the Tevatron," CTP-TAMU 13/87, NUB-2724, HUTP-87/A021, Jan 198\%. 8pp.

[25] J. L. Feng, M. E. Peskin, H. Murayama and X. Tata, Phys. Rev. D 52, 1418 (1995) arXiv:hep-ph/9502260.

[26] I. Hinchliffe, F. E. Paige, M. D. Shapiro, J. Soderqvist and W. Yao, Phys. Rev. D 55, 5520 (1997) arXiv:hep-ph/9610544.

[27] G. Polesello, L. Poggioli, E. Richter-Was, J. Soderqvist, Precision SUSY measurements with ATLAS for SUGRA point 5, ATLAS Internal Note, PHYS-No-111, October 1997.

[28] C. Diaconu, arXiv:hep-ph/0401111

[29] S. Dimopoulos and G. L. Landsberg, in Proc. of the APS/DPF/DPB Summer Study on the Future of Particle Physics (Snowmass 2001) ed. N. Graf, In the Proceedings of APS / DPF / DPB Summer Study on the Future of Particle Physics (Snowmass 2001), Snowmass, Colorado, 30 Jun - 21 Jul 2001, pp P321.

[30] C. M. Harris, P. Richardson and B. R. Webber, JHEP 0308, 033 (2003) arXiv:hep-ph/0307305.

[31] D. C. Dai, G. Starkman, D. Stojkovic, C. Issever, E. Rizvi and J. Tseng, arXiv:0711.3012] [hep-ph].

[32] K.S. Thorne, in: Magic without magic: John Archibald Wheeler, edited by J. Klauder (Freeman, San Francisco, 1972).

[33] V. Cardoso, E. Berti and M. Cavaglià, Class. Quant. Grav. 22, L61 (2005) arXiv:hep-ph/0505125.

[34] P. C. Aichelburg and R. U. Sexl, Gen. Rel. Grav. 2, 303 (1971).

[35] H. Yoshino and Y. Nambu, Phys. Rev. D 67, 024009 (2003) arXiv:gr-qc/0209003;

H. Yoshino and V. S. Rychkov, Phys. Rev. D 71, 104028 (2005) arXiv:hep-th/0503171].

[36] E. Berti, M. Cavaglià and L. Gualtieri, Phys. Rev. D 69, 124011 (2004) arXiv:hep-th/0309203.

[37] S. W. Hawking, Commun. Math. Phys. 43, 199 (1975) [Erratum-ibid. 46, 206 (1976)].

[38] R. Emparan, G. T. Horowitz and R. C. Myers, Phys. Rev. Lett. 85, 499 (2000) arXiv:hep-th/0003118;

M. Cavaglià, Phys. Lett. B 569, 7 (2003) arXiv:hep-ph/0305256.

[39] P. Kanti and J. March-Russell, Phys. Rev. D 66, 024023 (2002) arXiv:hep-ph/0203223;

P. Kanti and J. March-Russell, Phys. Rev. D 67, 104019 (2003) arXiv:hep-ph/0212199;

C. M. Harris and P. Kanti, JHEP 0310, 014 (2003) arXiv:hep-ph/0309054;

D. Ida, K. y. Oda and S. C. Park, Phys. Rev. D 67, 064025 (2003) [Erratum-ibid. D 69, 049901 (2004)] arXiv:hep-th/0212108;

A. S. Cornell, W. Naylor and M. Sasaki, JHEP 0602, 012 (2006) arXiv:hep-th/0510009.

[40] V. Cardoso, M. Cavaglià and L. Gualtieri, JHEP 0602, 021 (2006) arXiv:hep-th/0512116];

V. Cardoso, M. Cavaglià and L. Gualtieri, Phys. Rev. Lett. 96, 071301 (2006) [Erratum-ibid. 96, 219902 (2006)] arXiv:hep-th/0512002.

[41] B. Koch, M. Bleicher and S. Hossenfelder, JHEP 0510, 053 (2005) arXiv:hep-ph/0507138.

[42] S. Abdullin and F. Charles, Nucl. Phys. B 547, 60 (1999) arXiv:hep-ph/9811402;

M. Chiorboli, M. Galanti and A. Tricomi, Acta Phys. Polon. B 38, 559 (2007).

[43] P. Meade and L. Randall, arXiv:0708.3017 [hep-ph].

[44] http://pdg.lbl.gov/

[45] B. Abbott et al. [D0 Collaboration], Phys. Rev. Lett. 80, 2063 (1998) arXiv:hep-ex/9706014.

[46] H. Baer et al., arXiv:hep-ph/9503479 